$63^{\text {ème }}$ Congrès de la SFCO, 01002 (2015)

DOI:10.1051/sfco/20156301002

(C) Owned by the authors, published by EDP Sciences, 2015

\title{
CONFÉRENCE
}

\section{La chirurgie orthognatique en 2015}

\section{Bennaceur S}

CHU Robert Debré, Paris, France

selim.bennaceur@rdb.ap-hop-paris.fr

La chirurgie orthognathique actuelle repose sur les principes suivants : préparation orthodontique, chirurgie, puis finition orthodontique.

Il s'agit d'un traitement souvent long dans lequel le patient va passer plusieurs années. Ce principe pourtant fondateur de la chirurgie orthognathique des trente dernières années se voit de plus en plus écorné ; en effet, les progrès dans de la chirurgie comme dans l'orthodontie moderne associant les ancrages, la prévision chirurgicale par ordinateur, l'impression 3D... sont en train de modifier les protocoles de prise en charge. 